\title{
Photometric and dynamic evolution of an isolated disc galaxy simulation
}

\author{
L. Michel-Dansac ${ }^{1}$ and H. Wozniak ${ }^{1,2}$ \\ ${ }^{1}$ Observatoire de Marseille-Provence, Laboratoire d'Astrophysique de Marseille, 2 Place Le Verrier, \\ 13248 Marseille Cedex 4, France \\ 2 Present address: Centre de Recherche Astronomique de Lyon, 9 Av. Charles André, 69561 Saint-Genis Laval Cedex, France
}

Received 2 September 2003 / Accepted 20 March 2004

\begin{abstract}
We present a detailed analysis of the evolution of a simulated isolated disc galaxy. The simulation includes stars, gas, star formation and simple chemical yields. Stellar particles are split in two populations: the old one is present at the beginning of the simulation and is calibrated according to various ages and metallicities; the new population is born in the course of the simulation and inherits the metallicity of the gas particles. The result has been calibrated in four wavebands with the spectrophotometric evolutionary model GISSEL2000 (Bruzual \& Charlot 1993). Dust extinction has also been taken into account. A rest-frame morphological and bidimensional photometric analysis has been performed on simulated images, with the same tools as for observations. The effects of the stellar bar formation and the linked star formation episode on the global properties of the galaxy (mass and luminosity distribution, colours, isophotal radii) have been analysed. In particular, we have disentangled the effects of stellar evolution from dynamic evolution to explain the cause of the isophotal radii variations. We show that the dynamic properties (e.g. mass) of the area enclosed by any isophotal radius depends on the waveband and on the level of star formation activity. It is also shown that the bar isophotes remain thinner than mass isodensities a long time $(>0.7 \mathrm{Gyr})$ after the maximum of star formation rate. We show that bar ellipticity is very wavelength dependent as suggested by real observations. Effects of dust extinction on photometric and morphological measurements are systematically quantified. For instance, it is shown that, when the star formation rate is maximum, no more than $20 \%$ of the $B$ band luminosity can escape from the bar region whereas, without dust extinction, bar $B$ band luminosity accounts for $80 \%$ of the total $B$ band luminosity. Moreover, the extinction is not uniformly distributed inside the bar.
\end{abstract}

Key words. galaxies: evolution - galaxies: kinematics and dynamics - galaxies: spiral - galaxies: structure methods: $N$-body simulations

\section{Introduction}

Early $\mathrm{N}$-body models of isolated disc galaxies, using only collisionless particles, have clarified how several morphological features such as bars, spiral arms (e.g., Hohl 1978) and boxy bulges (Combes \& Sanders 1981) appear in stellar discs and evolve. Soon afterwards, it was realized that the role of the gas, the dissipative component, cannot be neglected. Collisionless $\mathrm{N}$-body codes were thus coupled with hydrodynamic codes, either for cosmological purposes (Katz \& Gunn 1991), or for detailed studies of isolated (Friedli \& Benz 1993) or merging galaxies (Mihos \& Hernquist 1996). For isolated disc galaxies, this kind of code has brought clear evidence that the presence of gas can deeply change the morphology on a short timescale (less than $1 \mathrm{Gyr}$ ). For instance, the gas can be responsible for the bar within bar phenomenon (Friedli \& Martinet 1993) or the dissolution of the bar and the formation of a bulge by secular evolution (Pfenniger \& Norman 1990; Norman et al. 1996).

Send offprint requests to: L. Michel-Dansac, e-mail: leo.michel-dansac@oamp.fr
Studies of the fueling mechanisms at work in central regions (e.g., Friedli \& Benz 1993; Shlosman \& Noguchi 1993) have been also studied with such hybrid codes.

However, stars and gas are not only bound together by gravitation, but also by the process of star formation and the energy and chemical feedback of supernovæ and stellar winds. Since star formation is not well understood even in our own Galaxy, several recipes have been implemented to mimic star formation in hydro+stellar dynamic codes. These recipes have allowed some authors to study for the first time the influence of bars on galactic abundance gradient (Friedli et al. 1994), the impact of the stellar ultraviolet radiation on star formation (Gerritsen \& Icke 1997), the nuclear activity (Heller \& Shlosman 1994), the starbursts induced by mergers (e.g., Mihos \& Hernquist 1996), the renewal of bars by gas accretion (Bournaud \& Combes 2002), etc. They also allow to perform self-consistent simulations of the formation and evolution of galaxies (e.g., Katz 1992; Steinmetz \& Muller 1995).

Whatever the kind of code used for the study of secular evolution, the morphological analysis of the simulations focuses 
on the properties of the mass distribution. To compare in detail these models with observations it is implicitly assumed that all particles have the same mass-to-light ratio taken as unity for convenience. However, the real galaxies are built of composite stellar populations, then of various mass-to-light ratios and metallicities. Thus, to make more straigthforward comparisons of simulations with multi-wavelength observations, we have used stellar population synthesis models to photometrically calibrate our self-consistent simulations which include stars, gas and star formation. We also take into account the effect of dust extinction.

This approach has been recently used to study the formation and the subsequent evolution of elliptical (e.g., Kawata 2001) or disc galaxies in a $\Lambda$ cold dark matter scenario (e.g., Westera et al. 2002; Abadi et al. 2003). Our own approach slightly differs from previous ones since we do not simulate the formation of a disc galaxy in a primeval Universe. Indeed, we restrict ourselves to a detailed study of the short-term evolution of an isolated disc galaxy already formed. All the particles are thus used to simulate the evolution of the galaxy.

We describe in Sect. 2 the numerical model and our technique of photometric calibration. The global evolution (i.e. integrated properties) of the model is presented in Sect. 3, and the morphological evolution in Sect. 4. We summarize our findings in Sect. 5.

\section{The $N$-body simulation and photometric calibration}

\subsection{Description of the code}

We used PMSPHSF, the $N$-body code developed in Geneva. It includes stars, gas and recipes to simulate star formation. The broad outlines of the code are the following: the gravitational forces are computed with a particle-mesh method using a 3D polar grid with $\left(N_{R}, N_{\phi}, N_{Z}\right)=(31,32,64)$ active cells (Pfenniger \& Friedli 1993), the hydrodynamics equations are solved using the SPH technique (Friedli \& Benz 1993). Star formation recipes are those implemented by Friedli \& Benz (1995). Here we briefly summarize the main features. For a detailed description of this modelling and a discussion on the influence of the free parameters, we refer the reader to Friedli \& Benz (1995).

Star formation and feedback modelling is based on the instantaneous star formation approximation. It consists of the following steps: the identification of the regions of star formation, the conversion of a fraction of gas into stars and the computation of the amount of energy and heavy elements injected in the interstellar medium (i.e. the energy and chemical feedback). Because the last step leads to the heating of gas, a simple treatment of radiative cooling is implemented in the energy equation.

The first task is to identify the gaseous particles that will form stars. Friedli \& Benz (1993) examined some possible criteria. Not surprisingly, they found that the standard Jeans instability criterion can be applied to spherical non-rotating gaseous systems, but that for rotating flat discs Toomre's instability criterion (Toomre 1964) is a better indicator. Observational evidence also appears to support the use of this criterion as a good indicator for locating star formation at intermediate scalelengths (Kennicutt 1989, 1990).

A particle $i$ will be assumed to undergo a star formation episode if the following condition is verified:

$Q_{i}^{\mathrm{g}}=\frac{s_{i} \kappa_{i}}{\pi G \Sigma_{i}^{\mathrm{g}}} \leq \lambda$

where $Q_{i}^{\mathrm{g}}$ is Toomre's parameter, $s_{i}$ is the local sound speed, $\kappa_{i}$ is the generalised epicyclic frequency, $\Sigma_{i}^{\mathrm{g}}$ is the gas surface density. This condition is clearly Toomre's stability criterion. The constant $\lambda$ equals unity in the case of an axisymmetric gaseous disc subject to radial instabilities (cf. Toomre 1964). However, a value of $\lambda \approx 1.4$ (with $s=6 \mathrm{~km} \mathrm{~s}^{-1}$ ) is derived from observations (Kennicutt 1990).

Once a particle of mass $m_{i}^{\mathrm{g}}$ subject to star formation has been identified, a fraction of its mass is converted into a stellar particle while total energy and momentum are conserved. The fraction of the gas particle actually transformed (i.e. the star formation efficiency factor $\epsilon$ ) is a free parameter. Note that within the framework of instanteneous star formation, $\epsilon$ is the product between the fraction of gas actually transformed into star $\eta$ (in accord with the initial mass function (IMF)) and the mass fraction $1-R$ finally blocked in low mass stars or stellar remnants. Thus, we can write $\epsilon=(1-R) \eta$. We re-use values of Friedli \& Benz (1995) $R \approx 0.45$ (Maeder 1992) and $0.02 \leq \eta \leq 0.5$. This translates into a possible range $0.01 \leq \epsilon \leq 0.25$. For the present work, $\epsilon=0.1$.

In practice, a particle $i$ subject to star formation is split into one star particle $m_{k}^{\star \text {,new }}$ and one gas particle $m_{i}^{\mathrm{g} \text {,new }}$ of reduced mass. Mass conservation requires that these masses be given by

$m_{k}^{\star \text {,new }}=\epsilon m_{i}^{\mathrm{g}}, \quad m_{i}^{\mathrm{g} \text {,new }}=(1-\epsilon) m_{i}^{\mathrm{g}}$.

At this stage, note that no Schmidt law (Schmidt 1959) has been imposed; the star formation rate (SFR) is thus a consequence of the (hydro)dynamic instabilities (see Sect. 5.2 of Friedli \& Benz 1995 for a discussion on the relation between the SFR and the gas surface density). The system can make several generations of stars from one gas particle and can thus quickly adjust itself to stabilise any collapsing region. However, to avoid numerical problems associated with the existence of gas particles differing widely in mass we restrict the allowable gas particle mass range to a maximum of 20. For $\epsilon=0.1$, this corresponds to a maximum of 29 generations of new stars from a single gas particle. Gas particles that reach the lower mass limit are transformed into a single collisionless particle.

Probably the most important effect of star formation on the global evolution of a galaxy is connected to the large amount of energy released in supernova explosions and stellar winds. In this modelling of feedback, the contribution of SNIa supernovæ is neglected. Let $E_{i, \text { tot }}^{\mathrm{SN}}$ be the total energy produced by SNII explosions at particle $i$ at a given timestep

$E_{i, \mathrm{tot}}^{\mathrm{SN}}=E_{i, \mathrm{~h}}^{\mathrm{SN}}+E_{i, \mathrm{mec}}^{\mathrm{SN}}=R^{\mathrm{SN}} \bar{E}^{\mathrm{SN}}(1-R)^{-1} m_{k}^{\star, \text { new }}$,

where $E_{i, \text { th }}^{\mathrm{SN}}=(1-f) E_{i, \text { tot }}^{\mathrm{SN}}$ and $E_{i, \mathrm{mec}}^{\mathrm{SN}}=f E_{i, \text { tot }}^{\mathrm{SN}}$ are respectively the thermal and mechanical energy injected, $R^{\mathrm{SN}}$ is the number 
of $\mathrm{SN}$ created per unit mass of stars formed, $\bar{E}^{\mathrm{SN}} \approx 10^{51} \mathrm{erg}$ is the mean energy produced by one SNII, and $f$ is a very poorly determined free parameter between 0 and 1 . It can be seen as the transfer efficiency of mechanical energy by thermalization to the surrounding ISM. For the present work, we use $f=0.01$ and $R^{\mathrm{SN}}=0.004$.

Depending on the model assumptions ( $Z$, IMF, SFR), the asymptotic value of energy ratio $R^{\mathrm{W}}$ between hot winds and SNII contributions can vary from almost 0 to about 2 (Leitherer et al. 1992). In particular, an increase of metals strongly increases $R^{\mathrm{W}}$. So, the contribution of winds to the energy injection is simply assumed to be proportional to the contribution coming from $\mathrm{SNe}$ but with a linear metallicity dependence, i.e. $E_{i, \text { tot }}^{\mathrm{W}}=R^{\mathrm{W}} E_{i, \mathrm{tot}}^{\mathrm{SN}}$ with $R^{\mathrm{W}}=25 Z$.

Energy, in both forms, is injected instantaneously and is spread over the $n_{i}$ neighbour particles $j$ of particle $i$ using the SPH smoothing kernel as the normalised weighting function $W_{i j}$. The fraction of mechanical energy given to particle $j$, $W_{i j}\left(E_{i, \mathrm{mec}}^{\mathrm{SN}}+E_{i, \mathrm{mec}}^{\mathrm{W}}\right)$, is added by computing the corresponding velocity vector increment

$(\Delta \boldsymbol{v})_{j}^{\mathrm{SN}}=\sqrt{\frac{2 W_{i j} f\left(1+R^{\mathrm{W}}\right) E_{i, \mathrm{tot}}^{\mathrm{SN}}}{m_{j}}} \frac{\boldsymbol{r}_{j}-\boldsymbol{r}_{i}}{\left|\boldsymbol{r}_{j}-\boldsymbol{r}_{i}\right|}, j=1, \ldots, n_{i}$.

Similarly, the specific thermal energy $E_{i, \text { th }}^{\mathrm{SN}}$ increment received by particle $j$ is given by

$\Delta u_{j}^{\mathrm{SN}}=\frac{W_{i j}(1-f)\left(1+R^{\mathrm{W}}\right) E_{i, \mathrm{tot}}^{\mathrm{SN}}}{m_{j}}, \quad j=1, \ldots, n_{i}$.

Since the instantaneous star formation approximation is used, instantaneous recycling is assumed as well. Therefore, during evolution, gas particles will see their abundances modified as star formation proceeds in either immediate neighbourhood, whereas star particles will be endowed at birth with a relative abundance pattern corresponding to their parent gas cloud. Changes of star abundance due to their evolution are neglected. Formally, the abundance change of a gas particle over one timestep can be written as

$Z_{j}^{\mathrm{g}}(t+\Delta t)=Z_{j}^{\mathrm{g}}(t)+\frac{y_{Z} m_{k}^{\star \text { new }} W_{i j}}{m_{j}^{\mathrm{g}}}, \quad j=1 \ldots n_{i}, i$,

where $W_{i j}$ is the weighting factor described previously, and $y_{Z}$ is the net yields for metals (i.e. the mass of metals ejected by all stars per unit mass locked into stars). The net yields are taken from Maeder (1992, model C, Table 7). At birth, star particles are created from gas, thus they have the same chemical composition.

The method of treating radiation losses is to use tabulated cooling functions from published data and to interpolate between them. For the present work, we use those of Boehringer $\&$ Hensler (1989) They cover the temperature range $10^{4} \mathrm{~K}$ to $10^{8} \mathrm{~K}$. Therefore, we assume a lower cut-off of $T_{\text {cut }}=10^{4} \mathrm{~K}$. Since we are not in a position to compute accurately the evolution of all elements, we restrict ourselves to cooling functions assuming solar abundance.

\subsection{Initial conditions}

An initial stellar population is set up to reproduce a disc galaxy with an already formed bulge. These particles form what we call hereafter the "old population" as opposed to particles created during the evolution ("new population").

The initial stellar positions for 500000 particles are drawn from a superposition of two axisymmetric Miyamoto-Nagai discs of mass respectively $10^{10}$ and $10^{11} M_{\odot}$, of scale lengths respectively 1.5 and $4 \mathrm{kpc}$ and common scale height of $0.5 \mathrm{kpc}$. The initial disc radius is $25 \mathrm{kpc}$.

Stellar velocities and velocity dispersions are found by solving numerically the Jeans equations directly on the grid using an iterative process. The initial conditions represent an equilibrium solution of the stellar hydrodynamics equations, incorporating the softened gravitation as well as the natural anisotropic velocity dispersion tensor of disc. This obviously does not mean that the stellar disc is fully stable. Indeed, the disc is dynamically cold enough to be bar mode unstable.

The gaseous component is represented by 50000 particles drawn from a third Miyamoto-Nagai disc of scale length of $6 \mathrm{kpc}$, scale height of $0.1 \mathrm{kpc}$ and truncation radius of $25 \mathrm{kpc}$. The total gas mass amounts to $1.1 \times 10^{10} M_{\odot}$. An initial gradient of metallicity has been imposed following the relationship $Z=0.02 \times \exp \left(-r / r_{Z}\right)$ where $r_{Z}$, the scalelength of the metallicity distribution, is $4.34 \mathrm{kpc}$. There is no vertical gradient as the gaseous component is initially distributed in a thin layer (scale height of $0.1 \mathrm{kpc}$ ).

We also ran the same simulation with a factor of 5 fewer particles to check whether the SFR and its variation in time depends on the number of particles. We find that our results reported in Sect. 3.2 are robust although the details of the dynamic evolution are obviously different.

For this first paper, we did not include any dark halo to be able in the near future to compare the effects of having added such an additional component. These initial conditions are identical to the Run A of Wozniak et al. (2003).

After 1 Gyr, the simulation numbers 1264729 particles, of which 26573 are gas particles. In comparison, the stellar disc and the dark matter halo of the final galaxy of Abadi et al. (2003) is described by less than 166000 particles. The final galaxy of Samland \& Gerhart (2003) contains 614500 stellar particles at the end of the simulation embedded in a growing halo.

In Table 2 we give the masses of the various components (old and new populations, gas) for the initial time and after an evolution of 1 Gyr.

\subsection{Stellar mass-to-light ratio calibration}

The output of any $N$-body simulation is a set of positions and velocities for all particles. In general, particles have the same masses so it is not necessary to make any assumption on their mass-to-light ratio. Thus, the problem of luminosity calibration has been widely overlooked. However, because of star formation, the particles created during the run have various masses, ages and metallicities. The observable properties, which are mean quantities integrated along the line-of-sight (LOS) and 
Table 1. Calibration of the old population.

\begin{tabular}{ccc}
\hline \hline & Age & Metallicity \\
\hline Model A & 10.4 & 0.004 \\
Model B & 10.4 & 0.020 \\
Model C & 6.7 & 0.004 \\
Model D & 6.7 & 0.020 \\
\hline
\end{tabular}

Table 2. Masses (in $10^{10} M_{\odot}$ ) of the various population at $t=0$ and $t=1 \mathrm{Gyr}$.

\begin{tabular}{lrrrrr}
\hline \hline Population & \multicolumn{2}{c}{ Whole simulation } & \multicolumn{2}{c}{ Central kpc } \\
& $t=0$ & & $t=1$ & $t=0$ & $t=1$ \\
\hline Old stars & & 11. & & 1.04 & 1.8 \\
Gas & 1.1 & & 0.53 & 0.08 & 0.0011 \\
New stars & 0 & & 0.57 & 0 & 0.43 \\
Total mass & \multicolumn{2}{c}{12.1} & & 1.12 & 2.23 \\
\hline
\end{tabular}

over a spatial resolution element, must be weighted by the $l u$ minosity of the particles. Therefore, we need an accurate massto-light ratio to convert masses into luminosities in various photometric bands.

For each stellar particle, given its age and metallicity, the mass-to-light ratio $\Upsilon$ was obtained from a bi-linear interpolation into the tables of GISSEL2000 (Bruzual \& Charlot 1993), for a Salpeter IMF, with mass cut-off at 0.1 and $100 M_{\odot}$. Then, luminosity of the particle $i\left(L^{i}\right)$, in the waveband $X$, is computed according to its mass $\left(M^{i}\right)$, birth metallicity $\left(Z_{\text {born }}^{i}\right)$ and the time elapsed since its birth $\left(t-t_{\text {born }}^{i}\right)$ :

$L_{X}^{i}(t)=\frac{M^{i}}{\Upsilon_{X}\left(t-t_{\mathrm{born}}^{i}, Z_{\mathrm{born}}^{i}\right)}$.

It is noteworthy that the photometric calibration is not fully consistent with star formation and feedback modelling since the selected value of $R^{\mathrm{SN}}$ corresponds to a lower mass limit for SNII equal to $\sim 12 M_{\odot}$ whereas the standard value $8 M_{\odot}$ is used in GISSEL2000.

For the old population (present at the beginning of the simulation) we have assumed ages of 6.7 and 10.4 Gyr and metallicities of $Z=0.004$ and $Z=0.02$ (cf. Table 1). Obviously, this implicitly assumes that all the old stars were simultaneously born 6.7 or 10.4 Gyr before the beginning of the simulation. Assuming an observer located at $z=0$ at the end of the simulation ( $t=1 \mathrm{Gyr}$ ), these calibrations imply a redshift of formation of $z=1$ and $z=3$ for resp. models C-D and models A-B.

Global quantities (e.g. luminosities, mass-to-light ratios, etc.) and surface brightness distribution were computed in various colours. We choose to work with four widely used broad band filters to cover the wavelength domain from the visible to the near infrared, namely $B, R, H$, and $K$ in the Johnson's system of GISSEL2000.

\subsection{Dust extinction}

Dust extinction in $B, R, H$ and $K$ band is simulated in the simplest way, assuming a constant gas-to-dust ratio

$N(\mathrm{H} \mathrm{I}) / A_{V}=5.34 \times 10^{21} \mathrm{~cm}^{-2}$.
$A_{V}$ is then converted to $A_{X}$ where $X$ stands for any waveband, according to the standard interstellar extinction law from Rieke \& Lebofsky (1985). H I column density should thus be integrated along the line-of-sight for each stellar particle, as Westera et al. (2002) did. Our SPH kernel is defined by (Monaghan \& Lattanzio 1985):

$$
W(r, h)=\frac{1}{\pi h^{3}} \begin{cases}1-\frac{3 v^{2}}{2}+\frac{3 v^{3}}{4} & \text { if } 0 \leq v \leq 1 \\ \frac{(2-v)^{3}}{4} & \text { if } 1 \leq v \leq 2 \\ 0 & \text { otherwise }\end{cases}
$$

where $v=r / h$. The contribution of each particle to the density vanishes for $r>2 h$. Thus, for each stellar particle, we should have to draw up a list of gas particles of which the sphere of radius $2 h$ is crossed by the line-of-sight. The gas column density is then the sum of individual gas particle density weighted by the SPH kernel.

This technique is obviously more difficult and CPU time consuming than with a grid. Therefore, we have computed extinction in a grid with the same spatial resolution than our images and 11 slabs along the line-of-sight from $z=-6.62 \mathrm{kpc}$ to $z=+6.62 \mathrm{kpc}$. The slab limits are spaced in $\log (|z|)$ in order to get a better resolution near the equatorial plane where most of the gas is concentrated. Each slab absorbs the stellar luminosity behind it. For each slab, the gas density distribution is obtain by the convolution of particle positions by the kernel.

\section{Global evolution over $1 \mathrm{Gyr}$}

The simulation has been stopped at 1 Gyr for the following reasons:

1. Our simulation should be considered as a numerical experiment. Most of the physical assumptions and recipes are simpler than in most chemodynamic numerical codes that are used to make simulations over several Gyr. This means that their validity might be questionable on longer timescales (e.g. the use of Toomre $Q$ parameter).

2. The closed box assumption is unlikely to be valid over a longer timescale. Gas infall, satellite harassment and/or merging, etc. are some examples of external perturbation that invalidate the closed box approximation.

3. Since we do not add any dark halo (fixed or with live particles), we neglect the interactions between the disc and the halo. It is thus not reasonable to study the long term effect of the disc evolution without any dark matter.

\subsection{Dynamic evolution}

The initial disc quickly develops a typical strong bar and a spiral structure (Fig. 1) both in the stellar and the gaseous components. The gravity torques due to the bar and spiral structure drive the gas inwards and reorganize the mass distribution even for the old stellar population; this gas inflow occurs in a rather short timescale since the SFR peaks at $t=0.3 \mathrm{Gyr}$ (cf. Fig. 3). As expected, star formation also occurs along gaseous spiral arms. 

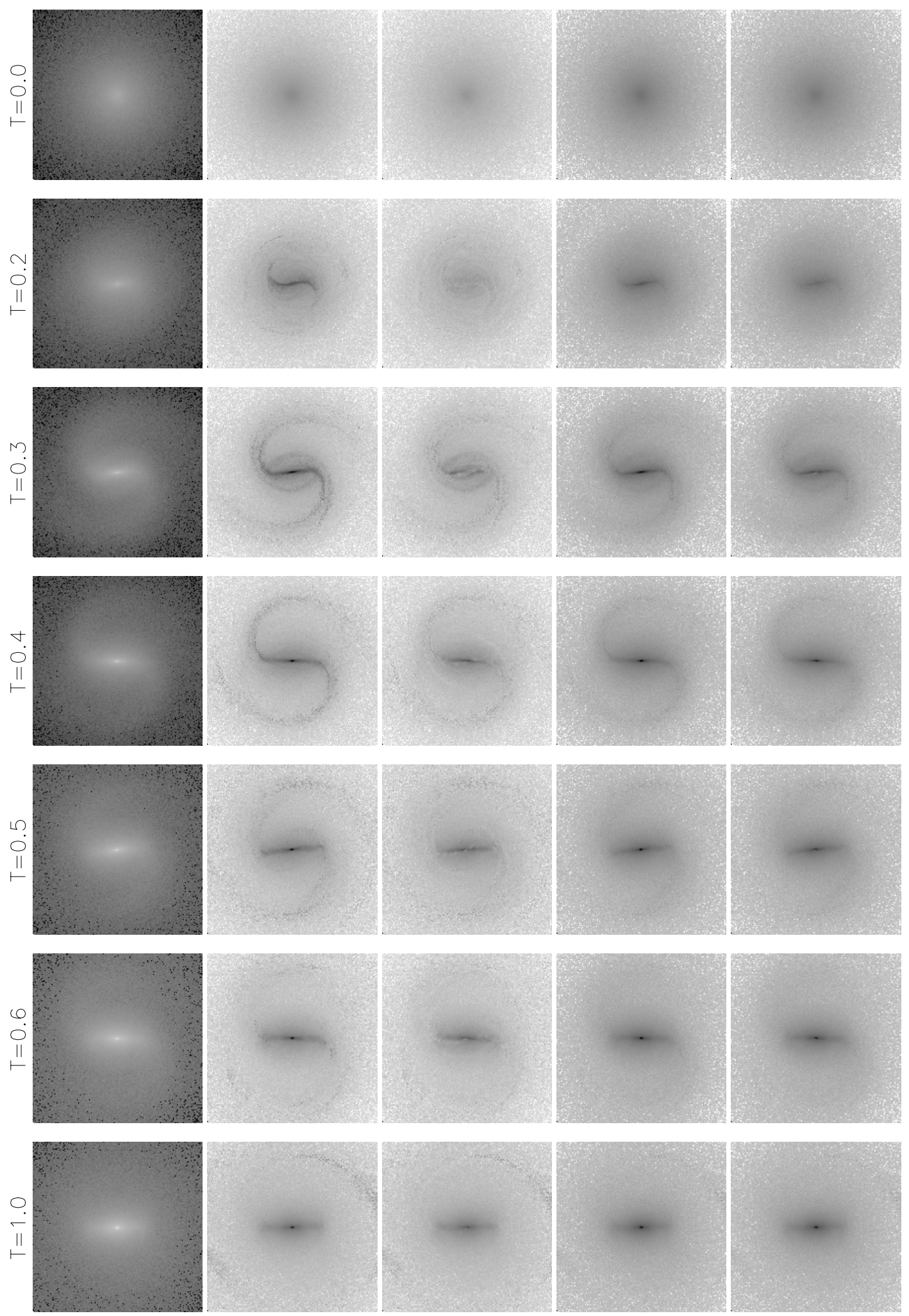

Fig. 1. Snapshots of the evolution of the simulation from $t=0$ to $t=1 \mathrm{Gyr}$. From left to right are displayed the mass distribution and $B$ dustfree, $B$ with extinction, $K$ dust-free, and $K$ with extinction calibrated images. The field of view $(20 \mathrm{kpc})$ is the same for each frame. The range in mass is $\left(1 ., 1.35 \times 10^{5}\right) M_{\odot} \mathrm{pc}^{-2}$, displayed in log units. $B$ surface brightness ranges from 12.75 to 28.8 mag $\operatorname{arcsec}^{-2}$ and $K$ ranges from 10.9 to $26.2 \mathrm{mag} \operatorname{arcsec}^{-2}$. The particles have been rotated so that the bar position is roughly horizontal.

After $1 \mathrm{Gyr}$, the total mass inside the central $\mathrm{kpc}$ has roughly doubled. Indeed, a nuclear gas disc is formed from the accumulation of gas in the centre, and new stars are actively formed there. The first cause of this mass inflow is the overall 
reorganization of the mass distribution under the influence of the stellar bar. Due to the gravitational torques exerted on the gas by the stellar bar, the extra mass in the form of gas and new stars amounts to $3.5 \times 10^{9} M_{\odot}$ at $t=1 \mathrm{Gyr}$, which is only $30 \%$ of the whole additional mass. Indeed, the old stars population contributes to the other $70 \%$.

The gas mass fraction inside the central kpc is very time dependent. For instance, between $t=0.905 \mathrm{Gyr}$ and $t=1 \mathrm{Gyr}$ the mass of gas is divided by a factor 4 . This illustrates a property of the gas inflow towards the central kpc region: the fueling is not stationary but rather proceeds in bursts.

Kinematical properties of the central kpc are described by Wozniak et al. (2003).

\subsection{Star formation rate evolution}

The SFR is displayed in Fig. 3. The newly formed stellar mass is averaged backward over two time windows of widths respectively $10 \mathrm{Myr}\left(S F R_{10}\right)$ and $100 \mathrm{Myr}\left(S F R_{100}\right)$. Apart from the first $S F R_{10}$ peak at $t=0.1 \mathrm{Gyr}$ which is due to the progressive switch-on of the star formation in the code, the other three $S F R_{10}$ maxima occurring around $0.3,0.5$ and $0.6 \mathrm{Gyr}$ are real. For $t \lesssim 0.3 \mathrm{Gyr}$, the bar and a bi-symmetric spiral structure spontaneously form. The gravitational torques applied by the bar and the spiral arms on the gas create several regions of very high gas density in which star formation is ignited. $S F R_{10}$ reaches $30 M_{\odot} \mathrm{yr}^{-1}$. As shown in previous works (e.g. Martin \& Friedli 1997), the star formation is not homogeneously distributed over the whole disc but is concentrated along the bar major axis and along the spiral arms (cf. Fig. 2). Indeed, 3/4 of the star formation occurs in the central $\mathrm{kpc}$ of the galaxy. SFR density ranges from $7.3 \times 10^{-4}$ (in the disc) to $365 M_{\odot} \mathrm{yr}^{-1} \mathrm{kpc}^{-2}$ (in the nucleus). These values are in good agreement with data from Kennicutt (1998).

The next two peaks at $t \approx 0.5$ and 0.6 Gyr are the result of the gas inflow towards the central region of the disc. Such inflow is not continuous, but rather proceeds in bursts.

When the $100 \mathrm{Myr}$ averaging window is used, the SFR maximum is moved forward by $\approx 50 \mathrm{Myr}$.

\subsection{Luminosity and colours evolution}

We plot in Fig. 4 the evolution of the integrated $B-H$ colour index. Our values are in good agreement with Scodeggio et al. (2002). Indeed, for their sample of spirals, $B-H$ values range between 1 and 4 . For the sake of comparison, two simple stellar populations (SSP) have been overplotted. One is the SSP of the old population and thus depends on the calibration model. The second is typical for a particle born at the maximum SFR ( $t=0.3 \mathrm{Gyr}$ ). Its metallicity is $Z_{\odot} / 2$.

In absence of dust, the galaxy becomes bluer by $\approx 1 \mathrm{mag}$ between $t=0$ and $t=0.25 \mathrm{Gyr}$, then $B-H$ slowly increases at a rate similar to the SSP of the population born at the maximum SSP. The colour of the disc is thus dominated by the population born during the most active phase of star formation around $t=0.3 \mathrm{Gyr}$.
When dust extinction is taken into account, the amplitude of the $B-H$ evolution is strongly smoothed. $B-H$ values remain close to the old population SSP. For models B and D, a $B-H$ minimum can still be determined but it is shifted towards $t \approx 0.4-0.5$ Gyr. For model $\mathrm{C}$, which has a young initial population with a low metallicity, $B-H$ does not display any clear minimum. Indeed, the colour remains roughly constant over 0.6 Gyr.

Figure 5a shows the total luminosity evolution for all the models without $\left(L_{\mathrm{tot}}\right)$ and with dust extinction $\left(L_{\mathrm{tot}}^{\mathrm{abs}}\right)$. Independently of the old population calibration, $L_{\mathrm{tot}}$ evolution clearly shows two regimes. The broad peak centred around $t=0.3$ Gyr obviously corresponds to the time when the star formation is the more active. There is a small but significant lag between the $L_{\text {tot }}$ peak in the $B$ and the infrared bands of $\approx 10 \mathrm{Myr}$ so that the $B$ peak is correlated to the $S F R_{10}$ maximum while the $K$ peak occurs between the $S F R_{10}$ and $S F R_{100}$ maximums. The maximum is followed by a slow decrease which corresponds to the dimming of the stellar population. However, the luminosity decrease is not exponential and is rather slower than for a SSP. This is due to a rate of star formation which remains high (above $2 M_{\odot} \mathrm{yr}^{-1}$ ) during the next $0.7 \mathrm{Gyr}$. This continuous star formation partly compensates for the ageing of the older stellar populations.

Hereafter we call luminosity contrast the ratio of the maximum value of the luminosity to the luminosity at $t=0$. For our calibration models, the latter corresponds to the luminosity of the old population at the beginning of the simulation, whereas the maximum value is the sum of luminosities of the old and of the new population.

The $L_{\text {tot }}$ contrast depends on the old population calibration, especially in the $B$ band. For instance, the $L_{\text {tot }}$ contrasts are typically $\approx 1.7$ in $K$ and between $\approx 3.8$ and $\approx 5.6$ in $B$ according to the calibration model. However, these values are not typical for a SSP since the contrast in the infrared is always higher than in $B$. For instance, the contrasts amount to $\approx 10$ in $K$ and $\approx 5$ in $B$ for a SSP with $Z=0.004$. This illustrates that the old population predominates in mass and that the $B$ band luminosity of the old population is $\approx 2$ decade lower than the $K$ band luminosity.

As expected, when dust extinction is taken into account, the total luminosities are fainter in all bands. However, since the amount of gas as well as its spatial distribution evolve because of the combined effects of the dynamic evolution and star formation, the ratio of unobscured to obscured luminosity also evolves with time (Fig. 5b).

It is also noteworthy that the luminosity peak at $t \approx 0.3 \mathrm{Gyr}$ is smoothed out in $R, H$ and $K$ band. In $B, L_{\text {tot }}^{\text {abs }}$ still displays a broad bump with a small maximum but shifted after $t>0.4$ Gyr. This delay is due to the fact that star formation with the highest SFR occurs in the regions with the highest gas density. In these regions, extinction is then the highest since we assume it is proportional to the gas column density. Only between $1 / 4$ and $1 / 3$ of $L_{\text {tot }}$ is able to escape from the galaxy at $t=0.3$ Gyr (cf. Fig. 5b). Thus, the regions which contain the youngest population and thus contribute the most to the $B$ band luminosity are also the most obscured (cf. Sect. 4.4.2). Since 

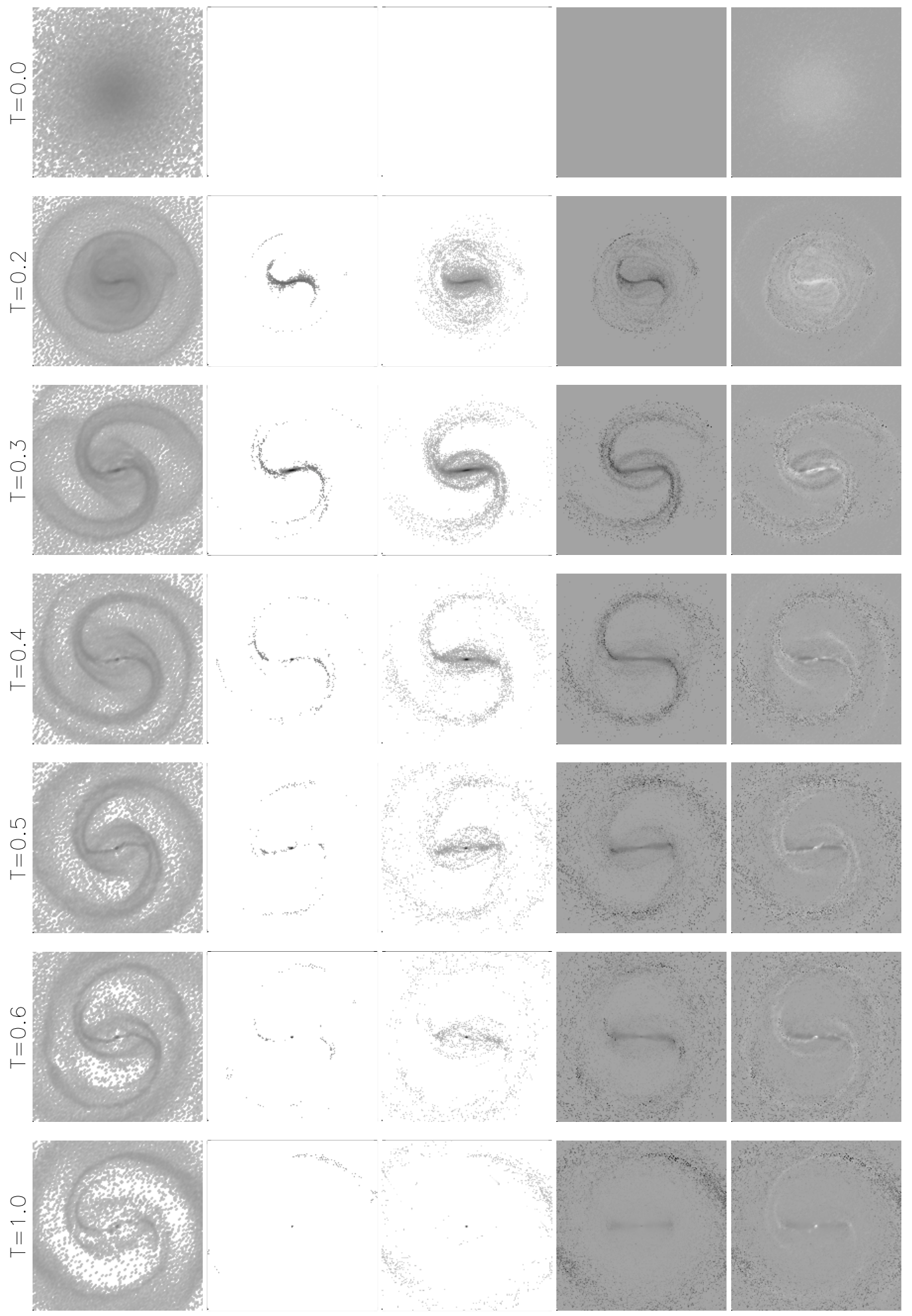

Fig. 2. Evolution of the simulation from $t=0$ to $t=1 \mathrm{Gyr}$. From left to right are displayed the gas mass distribution, the $S F R_{10}$ and $S F R_{100}$ distributions and $B-H$ colour map without and with dust extinction. The field of view $(20 \mathrm{kpc})$ is the same for each frame. For the gaseous mass density, a lower cutoff has been apply for the sake of clarity. The range in mass density is thus $\left(10^{-2}, 2.5 \times 10^{3}\right) M_{\odot} \mathrm{pc}^{-2}$, displayed in log units. $S F R_{10}$ and $S F R_{100}$ ranges from $7.3 \times 10^{-4}$ (in the disc) to $365 M_{\odot} \mathrm{yr}^{-1} \mathrm{kpc}^{-2}$ (in the nucleus), also displayed in log units. $B-H$ ranges from -0.57 (black) to 6.14 (white). The particles have been rotated so that the bar position is roughly horizontal.

a fraction of the gas amount is consumed during star formation and since gas and stars have different kinematics, the gas density decreases during the evolution. This enables the escape of the blue light after several tens of Myr. Consequently, the $L_{\text {tot }}^{\text {abs }}$ maximum should not be interpreted as a SFR maximum since it occurs $\sim 100$ Myr later on. 


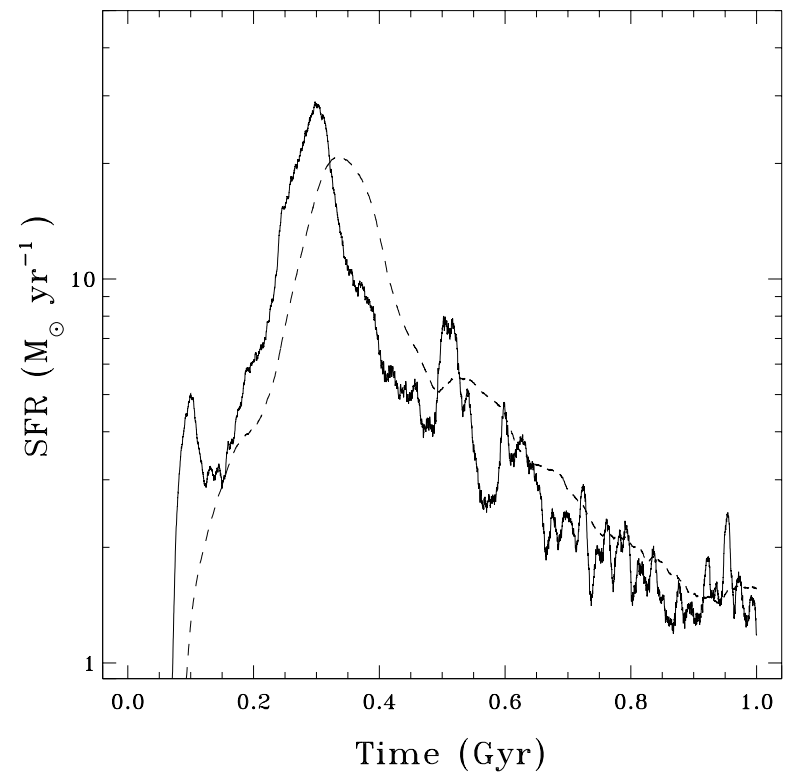

Fig. 3. Global star formation rate $\left(M_{\odot} \mathrm{yr}^{-1}\right)$ versus the elapsed time from the beginning of the simulation. Note that star formation is inhibited during the first $60 \mathrm{Myr}$. Backward time average windows of $10 \mathrm{Myr}$ (full line) and $100 \mathrm{Myr}$ (dashed line) has been used.

The high extinction of the youngest and brightest star forming regions also explains that the ratio $L_{\mathrm{tot}}^{\mathrm{abs}} / L_{\text {tot }}$ is sensitive to the parameters of the calibration model (cf. Fig. 5b) although the dust extinction model is exactly the same for all models. Indeed, since the $B$ band luminosity of young particles is absorbed by dust, the total $B$ band luminosity is dominated by the contribution of the old population. The differences between $L_{\text {tot }}$ and $L_{\mathrm{tot}}^{\text {abs }}$ are more marked in $B$ and $R$ than in the infrared bands, since differences in luminosity are more pronounced in $B$ and $R$ band than in infrared bands for the different old population. But the mean extinction in $K$ and $H$ band is not negligible since the ratio $L_{\text {tot }}^{\text {abs }} / L_{\text {tot }}$ amounts to 0.7 at $t=0.3 \mathrm{Gyr}$.

The stellar mass-to-light ratios are plotted in Fig. 6. Although the total stellar mass increases with respect to time because of star formation, the evolution of the total massto-light ratio is dominated by the total luminosity evolution. Values are in good agreement with mean values of the RC 3 catalogue for spiral type galaxies (Roberts \& Haynes 1994). Some observations (e.g., Vallejo et al. 2002) present lower values for mass-to-light ratio in $K$ band, indicating the presence of a very young population.

\subsection{Metallicity evolution}

Since an initial gradient of metallicity has been imposed on the gas particles at the beginning of the simulation (cf. Sect. 2.2), the metallicity of gas particles ranges from $6 \times 10^{-5}$ (at the disc edge) to 0.02 (at the centre). The mass-averaged metallicity $(\bar{Z})$ is $7.5 \times 10^{-3}$ (cf. Fig. 7). Then, $\bar{Z}$ and the maximum metallicity increase until $t=0.3 \mathrm{Gyr}$. During the same time, $\bar{Z}$ of the new population also increases and is always higher than for the gas since star formation is ignited in the central region of the disc. However, during the next $0.7 \mathrm{Gyr}$, the mass of the gas

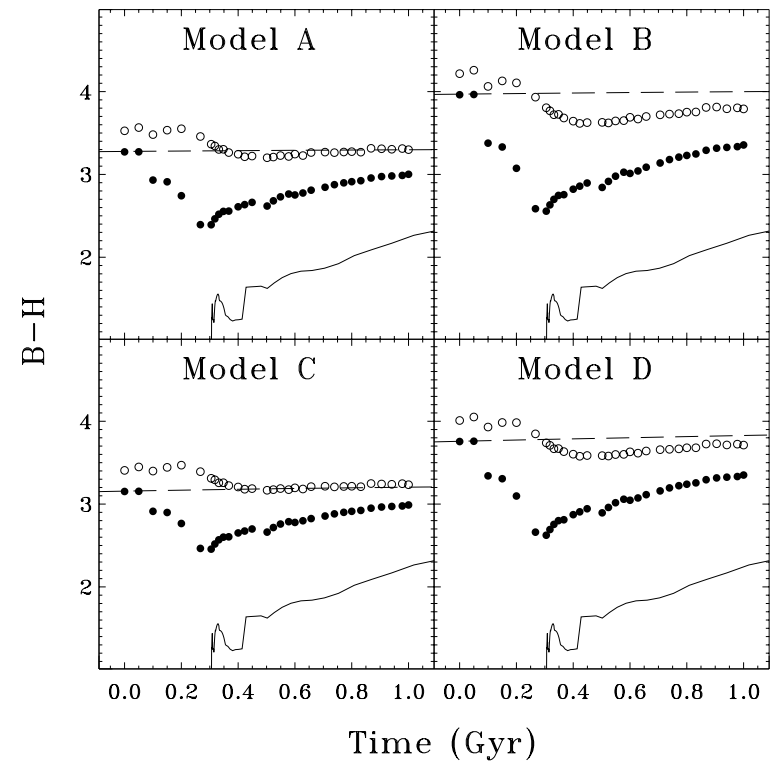

Fig. 4. $B-H$ colour evolution. Colours of the models without dust extinction are represented by full points, with extinction by empty points. The full line stands for the $B-H$ index of an SSP with the mean metallicity of gaseous star forming particles at $t=0.3 \mathrm{Gyr}$. The long-dashed curve is the $B-H$ colour of the old population.

significantly decreases because of the strong star forming rate $\left(S F R_{10} \approx 30 M_{\odot} \mathrm{yr}^{-1}\right)$. This implies that $\bar{Z}$ of the gas decreases while is remains constant for the new population. Some gas particles with a high metallicity are fully converted into new stars as their mass has reach the lower threshold. This explains that the maximum metallicity of the gas decreases.

At $t=1 \mathrm{Gyr}$, some low metallicity gaseous particles still exist, but because of the gas inflow towards the central region, their number decreases. When star formation becomes active for such particles (for instance when they encounter a spiral arm), they form new stars with a low metallicity, so that the minimum metallicity for the new population is a decreasing curve.

\section{Surface brightness evolution}

\subsection{Simulated images}

After individual particle calibration, simulated CCD images are obtained summing particle luminosities into a $512 \times 512$ pixels grid. The field of view is $60 \mathrm{kpc}$ which gives a spatial resolution of $\approx 117 \mathrm{pc}, 1.3$ times our smallest $N$-body grid resolution. We thus produce one frame per waveband and per snapshot of the simulation. Our results are obviously independent of the bar position-angle with respect to the North or any other axis. We thus decided to systematically rotate the positions of particles to align the bar with the $x$-axis. An example is displayed in Fig. 1 for model A.

Due to a low number of particles in the external parts of the disc, we have also build two times lower resolution images. In Fig. 8 we show the magnitude of the faintest pixel (in $B$ mag $\operatorname{arcsec}^{-2}$ ) that is reachable in a simulated image, depending on the calibration model. This value corresponds to pixels 

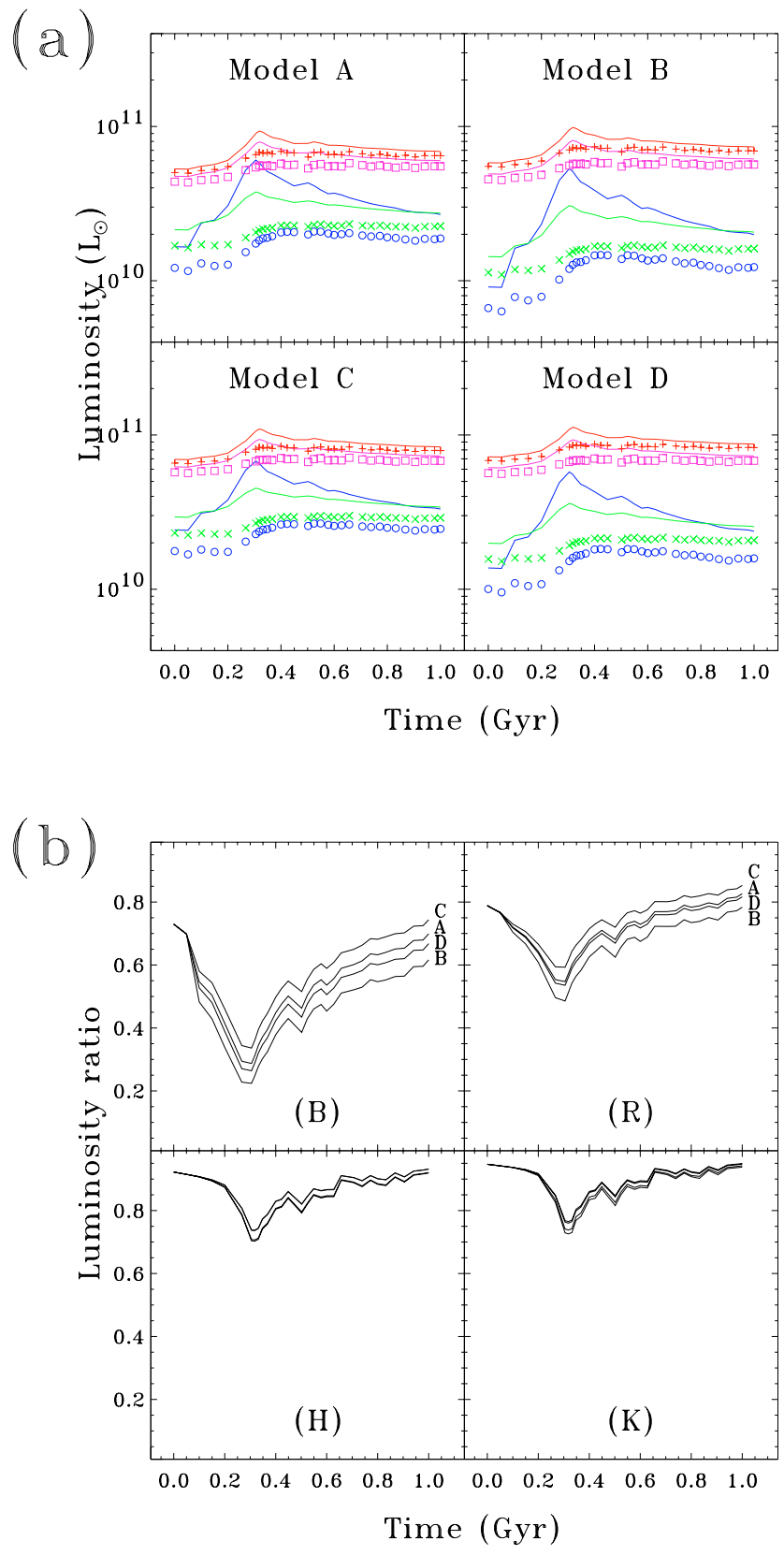

Fig. 5. a) Total luminosities for the four bands: $B$ (blue), $R$ (green), $H$ (magenta) and $K$ (red). Lines represent the total luminosity evolution of calibrated models without extinction $\left(L_{\mathrm{tot}}\right)$ while symbols stand for absorbed luminosities $\left(L_{\mathrm{tot}}^{\mathrm{abs}}\right)$. Metallicity of the old population increases from left $(Z=0.004)$ to the right $(Z=0.02)$, its age decreases from top (10.4 Gyr) to bottom $(6.7 \mathrm{Gyr})$. b) Evolution of the ratio $L_{\text {tot }}^{\text {abs }} / L_{\text {tot }}$ in $B$ (upper left panel), $R$ (upper right), $H$ (lower left) and $K$ (lower right). The letters beside the lines refer to the calibration model.

which contains only one particle. Because this only happens in the outskirts of the disc, where star formation is inactive, these isolated particles belong to the old population. This figure also show that our mass resolution for the old population $\left(2 \times 10^{5} M_{\odot}\right.$ per particle) disables the possibility to study calibration models with an initial age lower than $3.25 \mathrm{Gyr}$ if we expect to determine an isophotal diameter at the 25 th mag in the $B$ band.

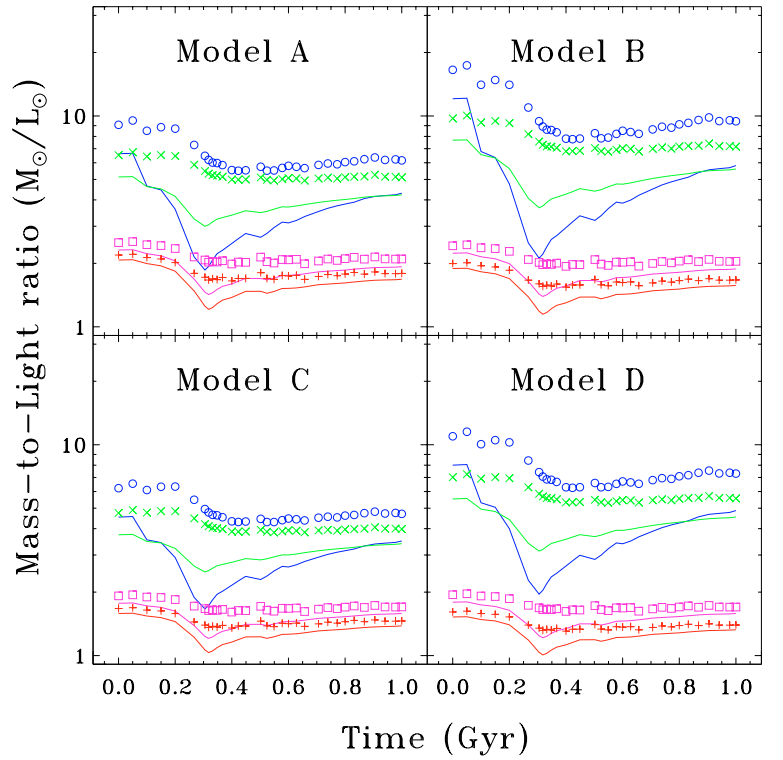

Fig. 6. Total mass-to-light ratio evolution. Lines, symbols and colours as for Fig. 5.

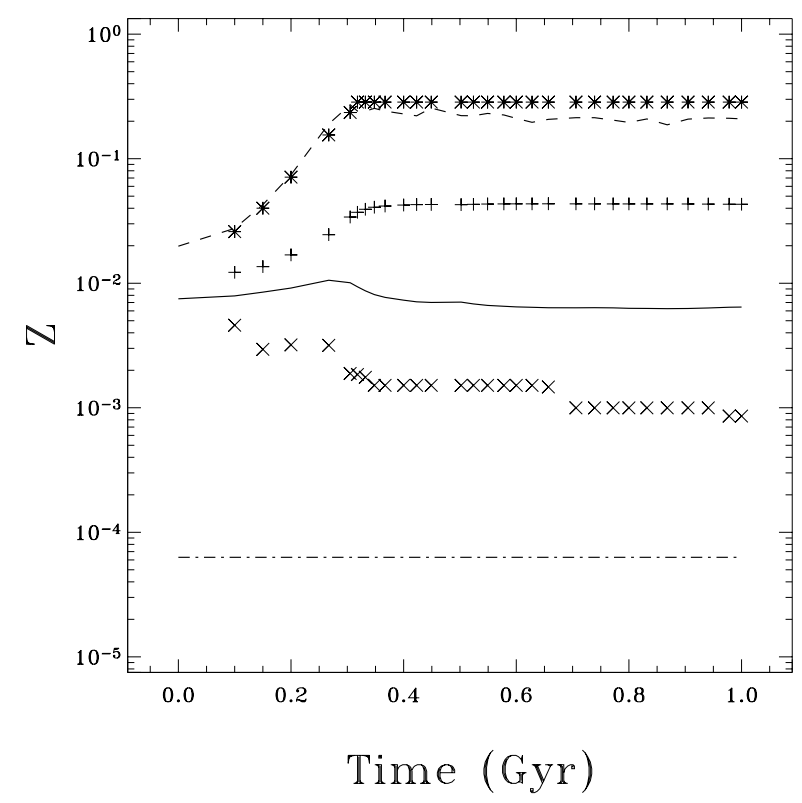

Fig. 7. Metallicity evolution of gaseous particles (lines) and new population particles (symbols). Mass-averaged (continuous line and pluses), minimum (dot-dashed line and crosses) and maximum metallicities (dashed line and stars) are drawn.

To mimic real observations we should convolve our images with a point spread function. However this last stage depends on the telescope and site characteristics. It thus introduces a few free parameters which cannot be constrained without any detailed comparisons with real observations.

In Fig. 2, $B-H$ colour maps are displayed for model A, as well as the mass surface density of the gaseous component and the $S F R_{10}$ and $S F R_{100}$ spatial distributions. In absence of dust extinction, $B-H$ colour maps and $S F R_{100}$ distribution are similar, which is no more the case when dust extinction is taken into account. The extinction is not uniformly distributed in the 


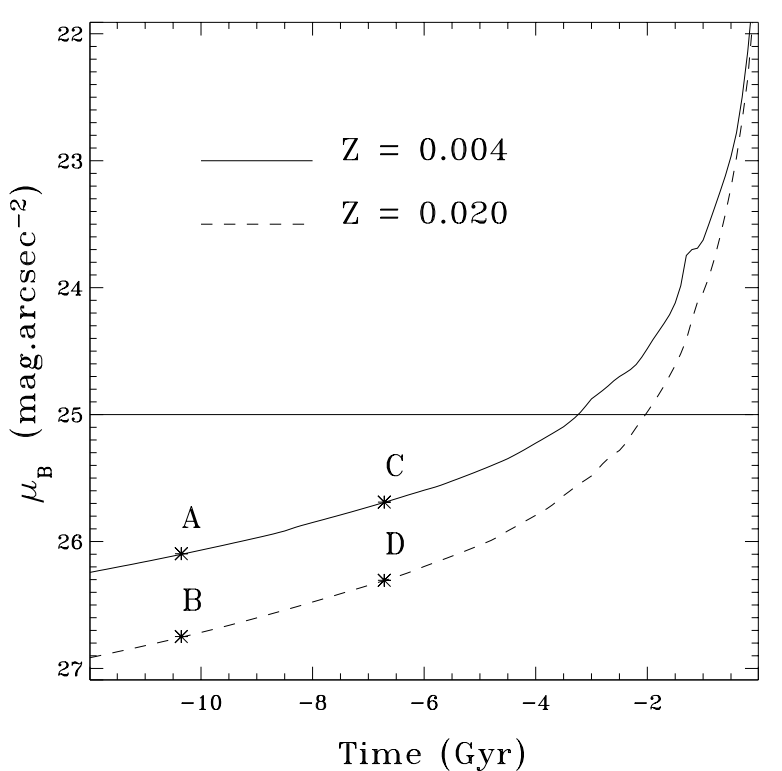

Fig. 8. $B$ band surface brightness of one particle of the old population isolated in a cell of a CCD-like image at $t=0$. The lines show the evolution of the surface brightness for the two metallicities used for calibration. The origin of the time is the beginning of the simulation $(t=0)$ so as the age of the old population is negative. The letters represent the model of calibration used for the old population (cf. Table 1). The 25th $B$ band magnitude is plotted as an horizontal line for reference.

bar region. It is obviously concentrated in the region of highest gas density and, therefore, in the region of high SFR.

\subsection{Ellipses fitting}

Each image has been analysed by fitting ellipses to the isophotes. This technique is very efficient in retrieving embedded structures (Wozniak et al. 1995; Friedli et al. 1996). In general, it also gives reliable structural parameters (Wozniak \& Pierce 1991). The algorithm used in the ellipse-fitting program is fully described in Jedrzejewski (1987). Thus, description of the procedure will be brief.

An isophotal contour of a given half-major axis length is written as:

$I(\varphi)=c_{0}+\sum_{n=1}^{\infty} c_{n} \cos (n \varphi)+d_{n} \sin (n \varphi)$.

If the trial ellipse exactly matches the isophotal contour all the coefficients but $c_{0}$ vanish. Then, $c_{0}$ is the mean intensity along the contour. The departure from exact ellipticity can be quantified with the Fourier components: errors in the position of the centre of the ellipse give non zero values for $c_{1}$ and $d_{1}$ while errors in ellipticity (defined by $e=1-b / a$ where $a$ and $b$ are respectively the half-major and half-minor axis lengths) and/or position-angle (PA) are revealed by $c_{2}$ and/or $d_{2}$ components.

Profiles of surface brightness, ellipticities and PA are obtained by increasing the half-major axis length $a$ by a factor 1.01 between each fit. We have chosen this small value to obtain a good resolution in the inner region.
The mean intensity profiles are then converted to surface brightness profiles. PA profiles follow the conventional notation. North is at the top, East to the left and the angles are counted in the anti-clockwise direction from $0^{\circ}$ to $180^{\circ}$. The bulk of the bar lies at $\mathrm{PA} \approx 0^{\circ}$. The range in radius for the figures begins at $1 \mathrm{px}$, i.e. $117 \mathrm{pc}$; the upper bound corresponds roughly to a surface brightness $\mu_{\mathrm{B}} \approx 10 \mathrm{mag} \operatorname{arcsec}^{-2}$. We show an example of these results in Fig. 9 for the model A at the same times than in Fig. 1.

\subsection{Isophotal radii}

The surface brightness profiles obtained with ellipse fitting has been used to determined the isophotal radius in each band. The surface brightness levels at which isophotal radii are measured are 25 in $B, 23.5$ in $R, 21.5$ in $K$ and 20.5 in $H$. For the $H$ band, we follow Gavazzi et al. (2000) while Gavazzi et al. (1996) used a limiting magnitude of 21.5.

Figure 10 displays the evolution of the isophotal radii for all the models. Apart from the $H$ band, the isophotal radii increase with time during the first $0.6 \mathrm{Gyr}$ and then remain constant or slightly decrease until $t=1 \mathrm{Gyr}$. In the $H$ band, $R_{20.5}^{H}$ clearly decreases after the SFR reaches its maximum value $(t=0.3 \mathrm{Gyr})$. The isophotal radius of a stellar disc, dynamically stable and free from star formation, should decrease with time since at a given radius the surface brightness increases with time. However, the disc of our simulation shows a strong dynamic evolution. We have shown in Sect. 3.1 that the mass is fully redistributed under the influence of the bar. The mass inside the central kpc doubles after $1 \mathrm{Gyr}$ and $70 \%$ of the additional mass come from the old population. Thus, the evolution of the isophotal radii could be the result of the combined effect of variations in luminosity due to star formation and stellar evolution, and the dynamic evolution. To disentangle the two effects, we plot in Fig. 11 the evolution of the fraction of mass inside the various isophotal radii and, in Fig. 12, the evolution of the fraction of luminosity inside isophotal radii.

The mass inside $R_{20.5}^{H}$ clearly decreases. However, the total $H$ band luminosity is rather constant or slowly increasing (Fig. 5a), while the fraction of luminosity inside $R_{20.5}^{H}$ drops from 80 to $65 \%$. Thus, the decrease of $R_{20.5}^{H}$ only reflects the dimming. It should be notice that $R_{20.5}^{H}$ is not a good estimator of the size of the galaxy since this radius only contains between 60 and $75 \%$ of the total mass and less than $80 \%$ of the total luminosity. The case of $R_{21.5}^{K}$ is similar although the mass decreases slower.

The evolution of the mass inside $R_{25}^{B}$ and $R_{23.5}^{R}$ is more tricky since it depends on the calibration of the old population. $R_{25}^{B}$ and $R_{23.5}^{R}$ are between 10 and $20 \%$ shorter when the metallicity of the old population is solar while they are only $10 \%$ shorter when the age of the old population is greater. These differences are roughly constant. Indeed, at these radii the old population dominates in mass and in luminosity. Thus, the differences in isophotal radii between models are directly linked to luminosity differences between old population. Moreover these difference are roughly constant over 1 Gyr for a many gigayears old population. 
$\mathrm{T}=0.2(\mathrm{Gyr})$

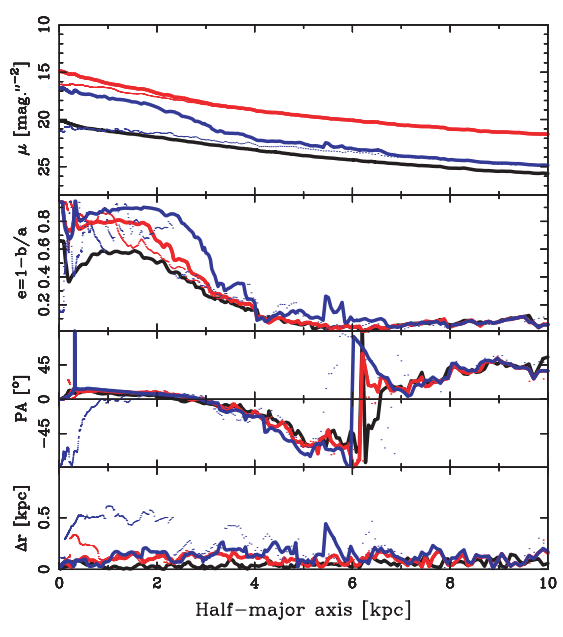

$\mathrm{T}=0.5(\mathrm{Gyr})$

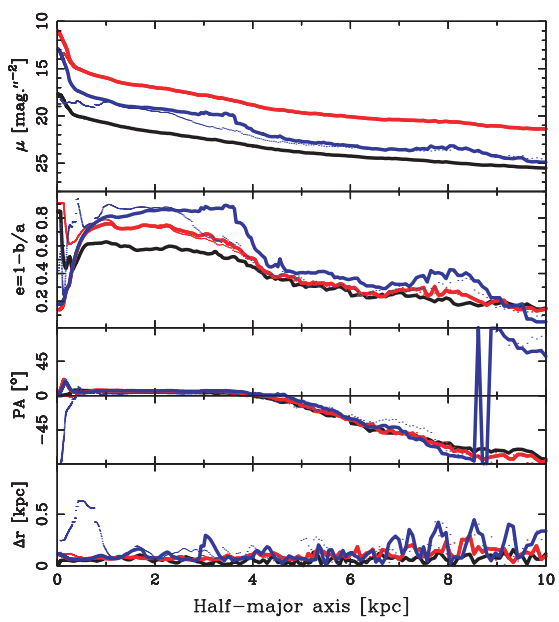

$\mathrm{T}=0.3(\mathrm{Gyr})$

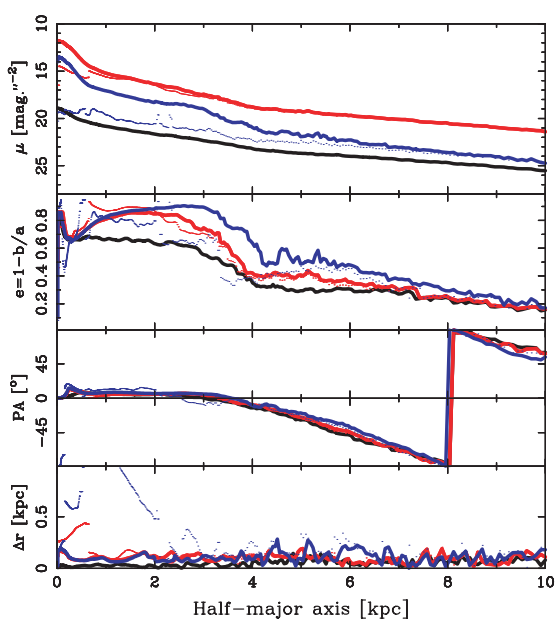

$\mathrm{T}=0.6(\mathrm{Gyr})$

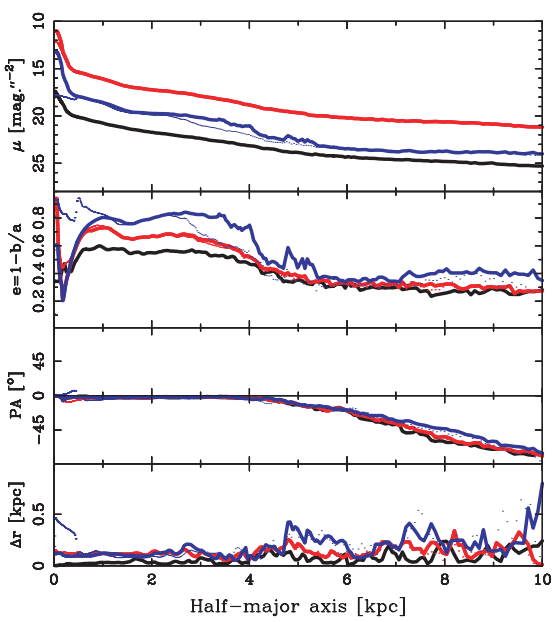

$\mathrm{T}=0.4(\mathrm{Gyr})$

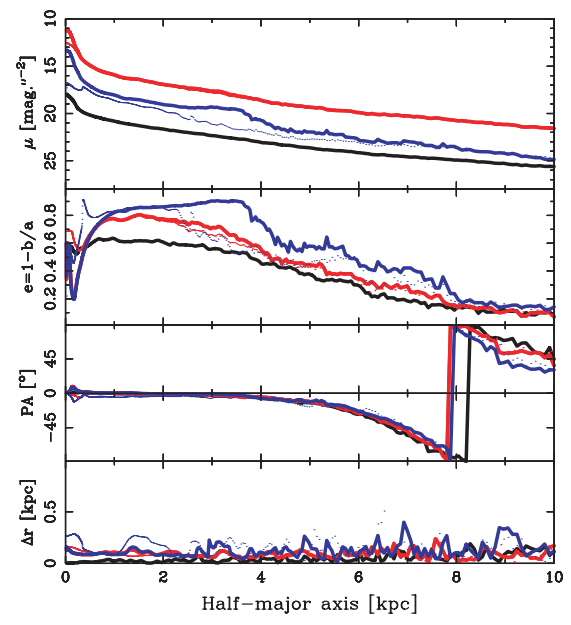

$\mathrm{T}=1.0(\mathrm{Gyr})$

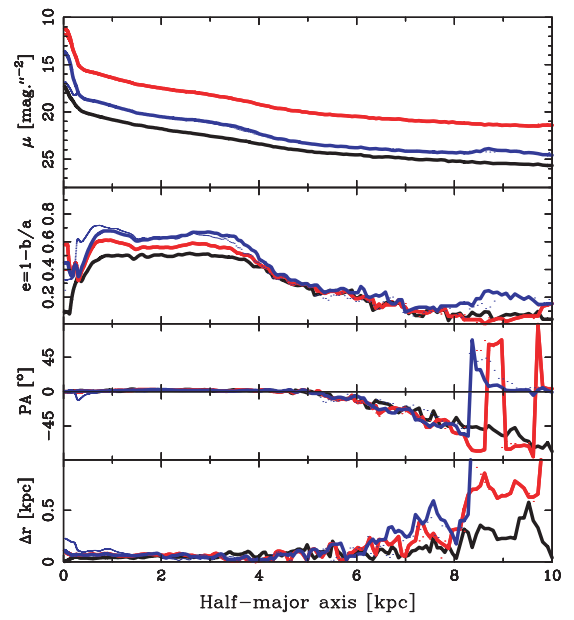

Fig. 9. For each panel: from top to bottom, surface brightness $(\mu)$, ellipticity $(e=1-b / a)$, position-angle (PA) and central shift for each fitted ellipse, for the two bands ( $B$ plotted in blue and $H$ in red) and the mass distribution (in black). The mass density profiles have been scaled so that the 30th mag corresponds to $1 M_{\odot} \mathrm{pc}^{-2}$. Results for dusty images are plotted in the form of dots.

It is also obvious from Fig. 10 that dust extinction is negligible at these radii since the gas density is very low. The measurements of isophotal radii are thus robust.

Finally, Fig. 12 also shows that the isophotal radii are not a consistent estimator of the size of a galaxy. Indeed, depending on the waveband and the delay with respect to the maximum SFR, these radii delineate regions of various properties (luminosity, mass, etc.) which cannot be compared between galaxies and, for the same object, between different wavelength range.

\subsection{Bar characteristics}

\subsubsection{Bar length and isophotal radii}

As displayed in Figs. 1 and 2, a stellar bar forms, grows and becomes rounder during the simulation. Thanks to our calibration, we are able to measure the bar length at various wavelengths. Several criteria, defined in the literature, can be used to determine the bar length. The results can then be compared with dynamic radii. This work will be published in a separate paper (Michel-Dansac \& Wozniak submitted). Hereafter, we will use bar lengths determined with a criterion shown to be in good agreement with the corotation radius (namely $\mathrm{C}_{3}$ in Michel-Dansac \& Wozniak, submitted). The bar lengths being similar in $H$ and $K$ band, we only display the results obtained from the $K$ band.

In Fig. 13, the evolution of the bar length to isophotal radius ratio is plotted. This ratio obviously increases during the bar formation phase then remains roughly constant and rather similar in both bands, for all the models apart from model B. Between $t \approx 0.3 \mathrm{Gyr}$ and $t \approx 0.4 \mathrm{Gyr}$ there is a peak.

It should be notice that the difference between models are mostly due to differences in the variation of the isophotal radius, since the length of the bar is almost independent of the calibration model.

The values are comparable to observational data from Martin (1995). For a sample of isolated barred galaxies (Martinet \& Friedli 1997), values of this ratio measured in $B$ band range between 0.04 and 0.47 . This ratio is insensitive to extinction effect since dust extinction does not corrupt measurements neither of the isophotal radii nor of the bar length. 


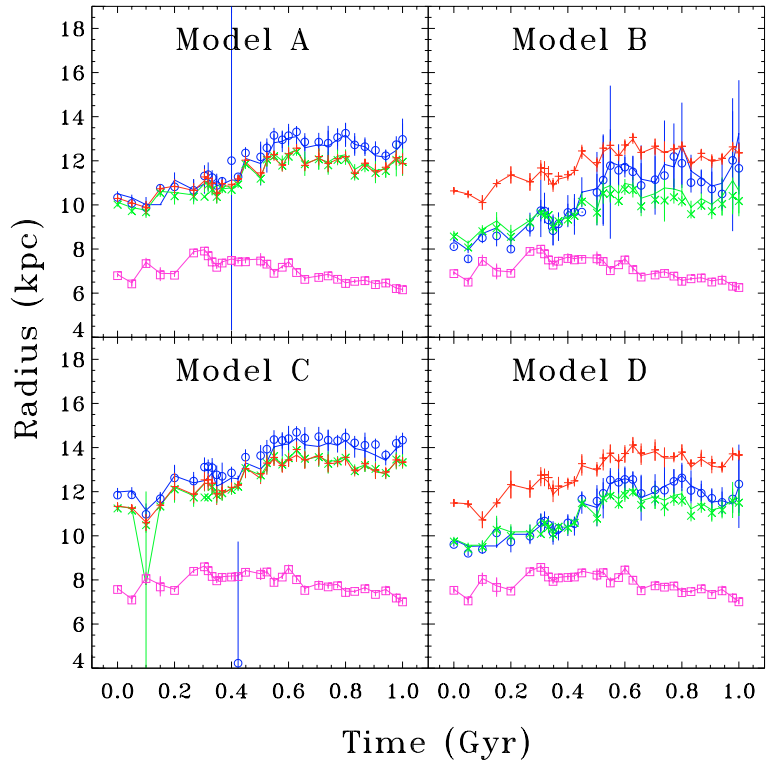

Fig. 10. Evolution of the isophotal radii: $R_{25}^{B}$ in $B$ (blue), $R_{23.5}^{R}$ in $R$ (green), $R_{20.5}^{H}$ in $H$ (magenta) and $R_{215}^{K}$ in $K$ (red). Symbols and lines as for Fig. 5.

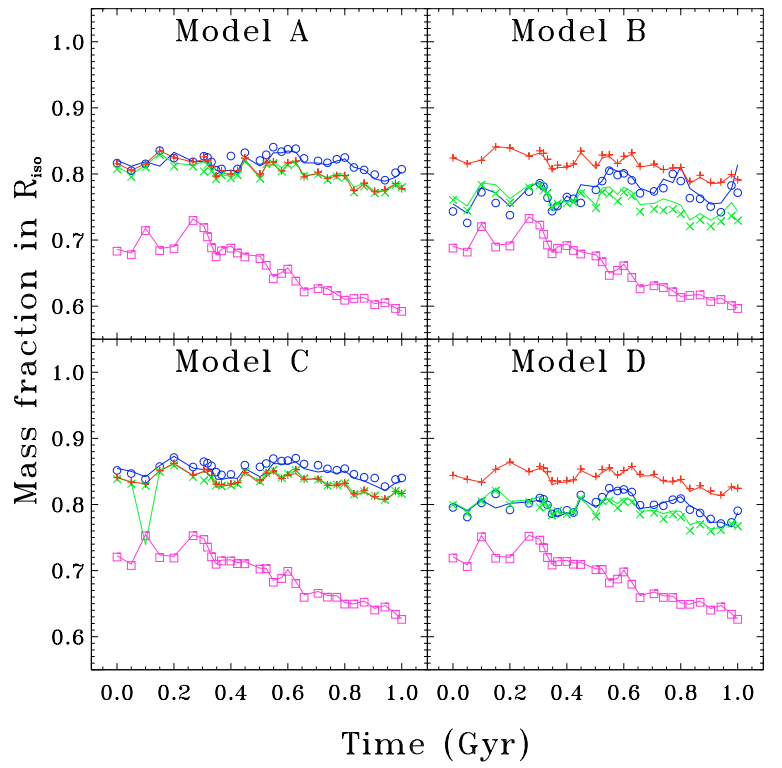

Fig. 11. Evolution of the fraction of mass inside the isophotal radii: $R_{25}^{B}$ in $B$ (blue), $R_{23.5}^{R}$ in $R$ (green), $R_{20.5}^{H}$ in $H$ (magenta) and $R_{21.5}^{K}$ in $K$ (red). Symbols and lines as for Fig. 5 .

\subsubsection{Luminosity of the bar region}

We have computed the total luminosity inside a circle of radius equal to the bar length.

Comparison of our value with the literature is not straightforward. Indeed, our definition of the bar luminosity includes the bulge and the nucleus. Moreover, since the criterion used to determine the end of the bar is a good tracer of the corotation (cf. Michel-Dansac \& Wozniak submitted), it gives upper limits of the bar lengths. In the case of real observations, the bulge and nucleus contributions are generally substracted and

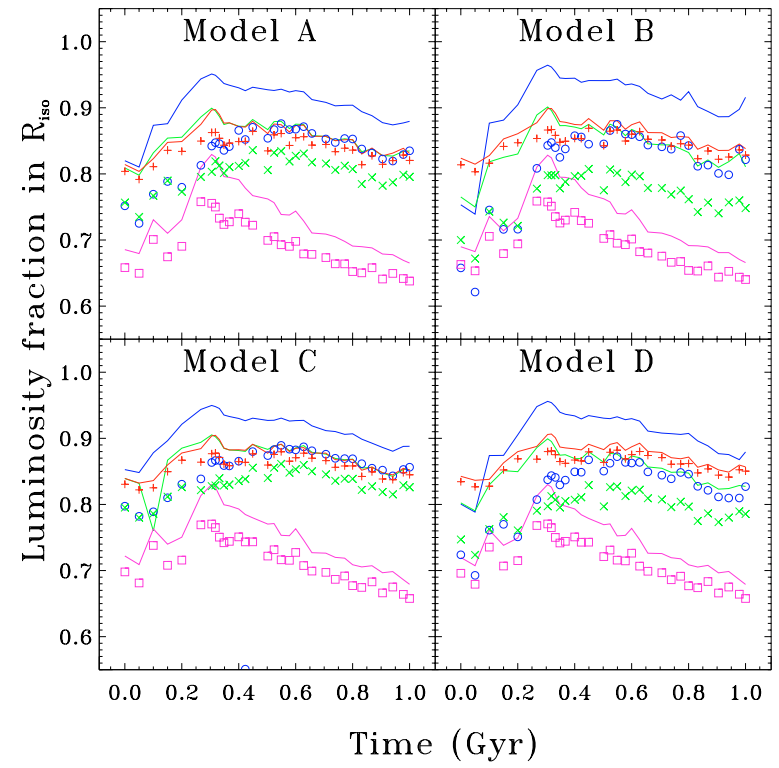

Fig. 12. Evolution of the fraction of luminosity integrated inside the isophotal radii: $R_{25}^{B}$ in $B$ (blue), $R_{23.5}^{R}$ in $R$ (green), $R_{20.5}^{H}$ in $H$ (magenta) and $R_{21.5}^{K}$ in $K$ (red). Symbols and lines as for Fig. 5 .

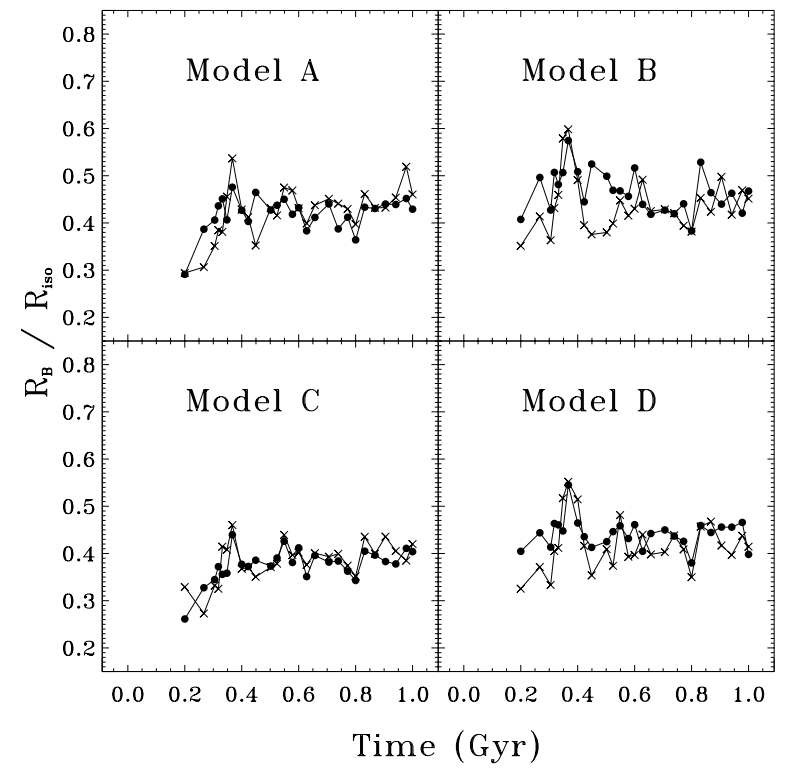

Fig. 13. Evolution of the bar length to isophotal radius ratio: $R_{25}^{B}$ in $B$ (full circles), $R_{21.5}^{K}$ in $K$ (crosses).

bars end before the corotation. Thus, our values of the bar luminosity represent upper limits.

Evolution of the bar luminosity to total luminosity ratio is plotted in Fig. 14 for the four models, in $B$ and $K$ band. In $B$ band, the ratio increases rapidly, reaches its maximum during the SFR peak, and then slowly decreases. A few weak bumps correspond to secondary SFR peaks. According to the model, $80 \%$ or more of the total $B$ band luminosity come from the bar region at the epoch of the peak of SFR. After 1 Gyr $(S F R<$ $2 M_{\odot} \mathrm{yr}^{-1}$ ) more than two third of the total blue luminosity still come from the bar region. Extinction effects in the bar region are important: at $t \approx 0.3$ Gyr less than $20 \%$ of the bar $B$ band 


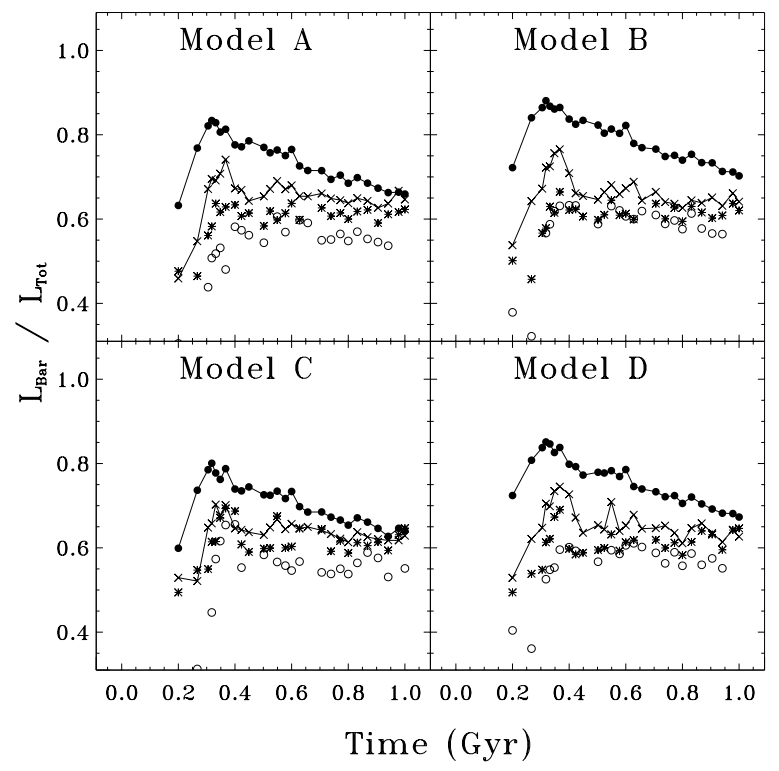

Fig. 14. Evolution of the bar luminosity to total luminosity ratio in $B$ (full circles) and $K$ (crosses). When extinction is taken into account, the ratio is plotted as open circles in $B$ and stars in $K$.

luminosity succeed to escape, and between 50 and $65 \%$ at $t \approx$ 1 Gyr according to the calibration models.

In the $K$ band, the ratio increases rapidly, reaches a maximum (70-75\%) during the SFR peak, and then rapidly (at $t \approx 0.4 \mathrm{Gyr}$ ) reaches an approximatively constant value of about two third of the total infrared luminosity. Extinction effects are almost negligible, just as model effects.

\subsubsection{Bar ellipticity}

Maximum ellipticities reached in the bar region are displayed in Fig. 15. Bars are significantly thinner in $B$ than in $K$ band, except for $t=0.27 \mathrm{Gyr}$. In the $B$ band, the bar is very thin until $t \approx 0.55$ Gyr. The maximum ellipticity then decreases with a slope which strongly depends on the calibration models. On the contrary, the $K$ band maximum ellipticity decreases immediatly after the SFR maximum. Hence, the difference between the two wavebands increases until $t \approx 0.6 \mathrm{Gyr}$, then it decreases with time but never vanishes.

Two causes are simultaneously responsible of this effect. Firstly, the spatial distribution of the new population is very elongated along the bar because new stars born in the gas flow along the bar which is narrower than the stellar bar (cf. Fig. 2). Then, secular dynamic diffusion is responsible for progressively making it rounder. This explains the high ellipticity reached in the bar when SFR is high and, only in part, the subsequent decrease. Secondly, the luminosity ratio between the new and the old populations is wavelength dependent, begin higher in $B$ than in $K$ band. Moreover, it roughly follows the SFR variations. The colour dependence slowly becomes blurred as SFR decreases. Hence, after the SFR peak, morphology becomes gradually dominated by the luminosity of the old population that has a rounder spatial distribution. This happens earlier in $K$ band. In $B$ band, the maximum ellipticity decreases

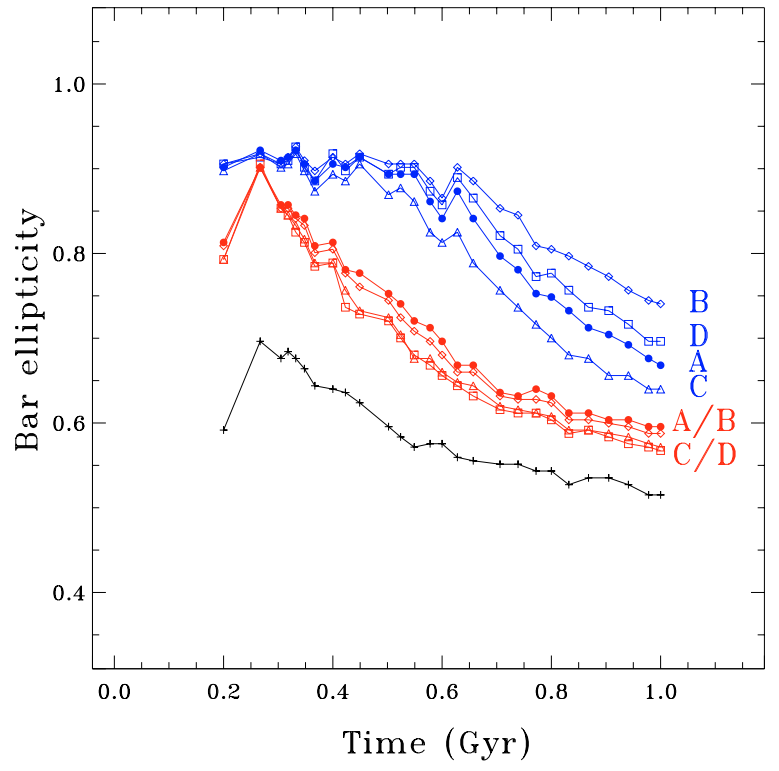

Fig. 15. Bar maximum ellipticity for all the models. Results for the mass distribution are displayed as black cross, red symbols for $K$ band images and blue symbols for $B$ band images. The letters beside the lines refer to the calibration model.

faster for more luminous old population (model C). This effect is weaker in $K$ band and is linked to the age rather than metallicity of the old population.

The maximum ellipticity of the mass distribution reaches its maximum at $t=0.27 \mathrm{Gyr}$, then slowly decreases. The formation of the bar and its thickening occurs in the old stellar population which dominates the mass. However, the mass distribution is always rounder than the luminosity one. Since difference decreases with SFR, it could be anticipated that isophotes and mass isodensities have the same shape if the SFR inside the bar vanishes. Thus, the surface brightness distribution, even in the near infrared, could be a good tracer of mass only if SFR remains low during a long time. Thus, mass modelling that used surface brightness distribution might give biased results if star formation is active, even at a moderate rate.

Dust extinction has obviously a more disturbing effect for $B$ isophotes than $K$ ones. Since extinction is proportional to $\mathrm{HI}$ column densities, it decreases with time following gas consumption by star formation. Ellipticity profiles are fully different and very noisy when extinction is high (cf. Fig. 9), making maximum ellipticity determination difficult. However, maximum ellipticiy values do not significantly differ from the dustfree case ${ }^{1}$. Differences on $B$ band ellipticity profile are still present at $t=1 \mathrm{Gyr}$ but appear mainly in the central kpc.

\section{Conclusions}

We have carried out a photometric calibration of a selfconsistent $\mathrm{N}$-body simulation of an isolated disc galaxy, including stars, gas and star formation. Dust extinction has been

1 An error occured in Fig. 2 of Michel-Dansac \& Wozniak (2003). In the left panel, we compare maximum ellipticity in $B$ band with and without extinction, whereas in the right panel, we compare maximum ellipticity in $B$ and $H$ band. 
taken into account. We have simultaneously studied the dynamic and photometric evolution in various wavebands $(B, R$, $H$ and $K$ ). We are able to reproduce observational properties such as mass-to-light ratio and $B-H$ colour indices. A morphological and photometric analysis have also been performed on simulated images in various wavebands, using the same tools as for real observations.

Our main results can be summarized as follows:

1. A dynamically self-consistent SFR peak produces a large increase in total luminosity in all wavebands whereas the stellar mass of the new population is much lower than the underlying old stellar mass. However, luminosity peaks can be almost completely obscured by dust extinction, since the young stellar population initially lies in gas-rich regions where extinction is maximum. This effect is particularly important in $B-H$ colour maps since regions of highest SFR could become unobservable. It could lead to underestimates of the SFR from photometric or colour measurements.

2. We show how isophotal radii evolve with respect to mass redistribution during the dynamic evolution but also with respect to stellar evolution. Hence, the dynamic properties of the area enclosed by any isophotal radius depends on the waveband and on the star formation activity. Extinction effects do not affect the determination of isophotal radii, even in $B$ band. It is also noteworthy that the luminosity integrated inside the isophotal radius at $20.5 \mathrm{mag} \mathrm{arcsec}-2$ in the $H$ band is not a good tracer of mass.

3. The luminosity integrated into the bar length reaches $\approx 80 \%$ of the total $B$ band luminosity at the SFR maximum in the absence of dust, and $\gtrsim 60 \%$ when the SFR is low, whereas the bar length remains smaller than or equal to half the isophotal radius.

4. When the SFR is high ( $\approx 30 M_{\odot} \mathrm{yr}^{-1}$ ) less than $20 \%$ of the $B$ band luminosity of the bar region escapes in the presence of dust because most star formation occurs in gas-rich regions where extinction is most efficient.

5. As long as star formation is active, mass isodensities of the bar region are rounder than isophotes. Thus, the surface brightness distribution, even in $H$ band, is a good tracer of mass only when the SFR is below $1 M_{\odot} \mathrm{yr}^{-1}$. Mass modelling that used the surface brightness distribution might give biased results if star formation is active, even at a moderate rate.

The use of other morphological classification tools (e.g. concentration and asymmetry, Abraham et al. 1996) will be reported in a forthcoming paper.

Acknowledgements. Our computations were partly performed on the Fujitsu NEC SX-5 hosted by IDRIS/CNRS. We are grateful to P. Berzcik and the anonymous referee for fruitful comments.

\section{References}

Abadi, M. G., Navarro, J. F., Steinmetz, M., \& Eke, V. R. 2003, ApJ, 591, 499

Abraham, R. G., Tanvir, N. R., Santiago, B. X., et al. 1996, MNRAS, 279, L47

Boehringer, H., \& Hensler, G. 1989, A\&A, 215, 147

Bournaud, F., \& Combes, F. 2002, A\&A, 392, 83

Bruzual, G., \& Charlot, S. 1993, ApJ, 405, 538

Combes, F., \& Sanders, R. H. 1981, A\&A, 96, 164

Friedli, D., \& Benz, W. 1993, A\&A, 268, 65

Friedli, D., \& Benz, W. 1995, A\&A, 301, 649

Friedli, D., Benz, W., \& Kennicutt, R. 1994, ApJ, 430, L105

Friedli, D., \& Martinet, L. 1993, A\&A, 277, 27

Friedli, D., Wozniak, H., Rieke, M., Martinet, L., \& Bratschi, P. 1996, A\&AS, 118, 461

Gerritsen, J. P. E., \& Icke, V. 1997, A\&A, 325, 972

Gavazzi, G., Pierini, D., Boselli, A., \& Tuffs, R. 1996, A\&AS, 120, 489

Gavazzi, G., Franzetti, P., Scodeggio, M., Boselli, A., \& Pierini, D. 2000, A\&A, 361, 863

Heller, C. H., \& Shlosman, I. 1994, ApJ, 424, 84

Hohl, F. 1978, ApJ, 83, 768

Jedrzejewski, R. I. 1987, MNRAS, 226, 747

Katz, N., \& Gunn, J. E. 1991, ApJ, 377, 365

Katz, N. 1992, ApJ, 391, 502

Kawata, D. 2001 ApJ, 558, 598

Kennicutt, R. C. 1989, ApJ, 344, 685

Kennicutt, R. C. 1990, The Interstellar Medium in Galaxies, ASSL, 161,405

Kennicutt, R. C. 1998, ARA\&A, 36, 189

Leitherer, C., Robert, C., \& Drissen, L. 1992, ApJ, 401, 596

Mihos, J. C., \& Hernquist, L. 1996, ApJ, 464, 641

Maeder, A. 1992, A\&A, 264, 105

Martin, P. 1995, AJ, 109, 2428

Martin, P., \& Friedli, D. 1997, A\&A, 326, 449

Martinet, L., \& Friedli, D. 1997, A\&A, 323, 363

Michel-Dansac, L., \& Wozniak, H. 2003, Ap\&SS, 284, 925

Monaghan, J. J., \& Lattanzio, J. C. 1985, A\&A, 149, 135

Norman, C. A., Sellwood, J. A., \& Hasan, H. 1996, ApJ, 462, 114

Pfenniger, D., \& Friedli, D. 1993, A\&A, 270, 561

Pfenniger, D., \& Norman, C. 1990, ApJ, 363, 391

Rieke, G. H., \& Lebofsky, M. J. 1985, ApJ, 288, 618

Roberts, M. S., \& Haynes, M. P. 1994, ARA\&A, 32, 115

Samland, M., \& Gerhard, O. 2003, A\&A, 399, 961

Schmidt, M. 1959, ApJ, 129, 243

Scodeggio, M., Gavazzi, G., Franzetti, P., et al. 2002, A\&A, 384, 812

Shlosman, I., \& Noguchi, M. 1993, ApJ, 414, 474

Steinmetz, M., \& Muller, E. 1995, MNRAS, 276, 549

Toomre, A. 1964, ApJ, 139, 1217

Vallejo, O., Braine, J., \& Baudry, A. 2002, A\&A, 387, 429

Westera, P., Samland, M., Buser, R., \& Gerhard, O. E. 2002, A\&A, 389, 761

Wozniak, H., \& Pierce, M. J. 1991, A\&AS, 88, 325

Wozniak, H., Combes, F., Emsellem, E., \& Friedli, D. 2003, A\&A, 409, 469

Wozniak, H., Friedli, D., Martinet, L., et al. 1995, A\&AS, 111, 115 